\title{
Numerical simulation of shock wave structure in nitrogen
}

Tatiana G. Elizarova

Institute of Mathematical Modeling, Russian Academy of Sciences, Miusskaya Square 4a, Moscow 125047, Russia

Anton A. Khokhlov

Faculty of Physics, M. V. Lomonosov Moscow State University, Leninskie Gory, Moscow 119992, Russia

Salvador Montero ${ }^{a)}$

Instituto de Estructura de la Materia, CSIC, Serrano 121, 28006 Madrid, Spain

(Received 31 January 2007; accepted 26 February 2007; published online 11 June 2007)

The one-dimensional problem of the structure of a stationary shock wave in nitrogen is solved in the frame of the Navier-Stokes (NS) equations. Proper interpretation of the bulk viscosity coefficient included in the shear stress tensor leads to a numerical solution close to the experiment, showing that the NS equations provide more accurate solutions to the problem than supposed previously.

(C) 2007 American Institute of Physics. [DOI: 10.1063/1.2738606]

The width and the density profile of one-dimensional stationary shock waves have often been employed as a test problem for numerical models of rarefied gas flows. Previous studies on argon, helium, and nitrogen shock waves have shown the difficulties and limitations of the Navier-Stokes (NS) equations. ${ }^{1-3}$ Experimental data for these studies are compiled in Ref. 4. Earlier calculations ${ }^{5,6}$ have shown substantial differences with the experiments for Mach numbers $\mathrm{Ma}>2$. This motivated several attempts towards improving NS modeling for rarefied flows, as shown in Ref. 7 and the literature in it.

In a recent work, ${ }^{8}$ it has been shown that argon shock waves up to $\mathrm{Ma}=10$ can be calculated in the NS approach with far better precision for the density profile than thought before, on the order of $30 \%$. Argon is, however, a monatomic species with no internal degrees of freedom. Diatomic molecules pose different problems due to the internal degrees of freedom.

In this Brief Communication, the one-dimensional shock wave problem is solved for molecular nitrogen, the results being compared with Alsmeyers compilation. ${ }^{4}$ Molecular nitrogen has one vibrational and two rotational degrees of freedom. The vibrational collision number being much larger than the rotational one ${ }^{9}$ suggests that vibration does not need to be taken into account here, to a good approximation. On the contrary, its comparatively low rotational collision number $Z \sim 5-16$ (Ref. 1) in the thermal range $300 \leqslant T$ $\leqslant 6000 \mathrm{~K}$ of shock waves up to $\mathrm{Ma}=10$ suggests a more efficient transfer of energy between rotational and translational degrees of freedom, which needs to be considered in the present problem. This contribution of the internal degrees of freedom to the shock wave problem is taken here into account by means of the bulk viscosity and its effect on the NS system.

A traditional shooting method to a matching point is employed for solving the steady-state NS system. This method,

${ }^{a)}$ Electronic mail: emsalvador@ iem.cfmac.csic.es with specific features for the boundary conditions, converges very fast. A relaxation technique previously employed for solving the NS system ${ }^{8}$ imposes two direct boundary conditions and delivers the same results, but converges about thousand times slower.

The NS equations for stationary one-dimensional plane flow read

$$
\begin{aligned}
& \frac{d(\rho u)}{d x}=0, \\
& \frac{d\left(\rho u^{2}\right)}{d x}+\frac{d p}{d x}=\frac{d \Pi}{d x}, \\
& \frac{d(\rho u H)}{d x}+\frac{d q}{d x}=\frac{d(\Pi u)}{d x},
\end{aligned}
$$

where $\rho$ stands for the gas density, $u$ for the velocity, and $p=\rho R T$ for the pressure at the temperature $T$, with $R$ is the gas constant; $H=(E+p) / \rho$ is the total enthalpy per unit volume, $E=\rho u^{2} / 2+p /(\gamma-1)$ is the total energy per unit volume, and $\gamma$ the specific heat ratio. The shear-stress tensor ( $\Pi)$ and the heat flux vector $(q)$ in Eqs. (2) and (3) are defined as

$$
\Pi=\left(\frac{4}{3} \eta+\xi\right) \frac{\partial u}{\partial x}, \quad q=-\kappa \frac{\partial T}{\partial x},
$$

where $\eta$ is the viscosity coefficient, $\xi$ the bulk viscosity, and $\kappa$ the heat conductivity coefficient; $\eta$ and $\kappa$ are related by

$$
\kappa=\frac{\gamma R}{(\gamma-1) \operatorname{Pr}} \eta,
$$

where $\mathrm{Pr}$ is the Prandtl number. The thermal dependence of the viscosity coefficient will be described by the function

$$
\eta=\eta_{\mathrm{ref}}\left(\frac{T}{T_{\mathrm{ref}}}\right)^{\omega}
$$

where $\eta_{\text {ref }}$ is the value of $\eta$ at the temperature $T_{\text {ref }} ; \omega$ is a constant, which depends on the nature of the gas considered. 


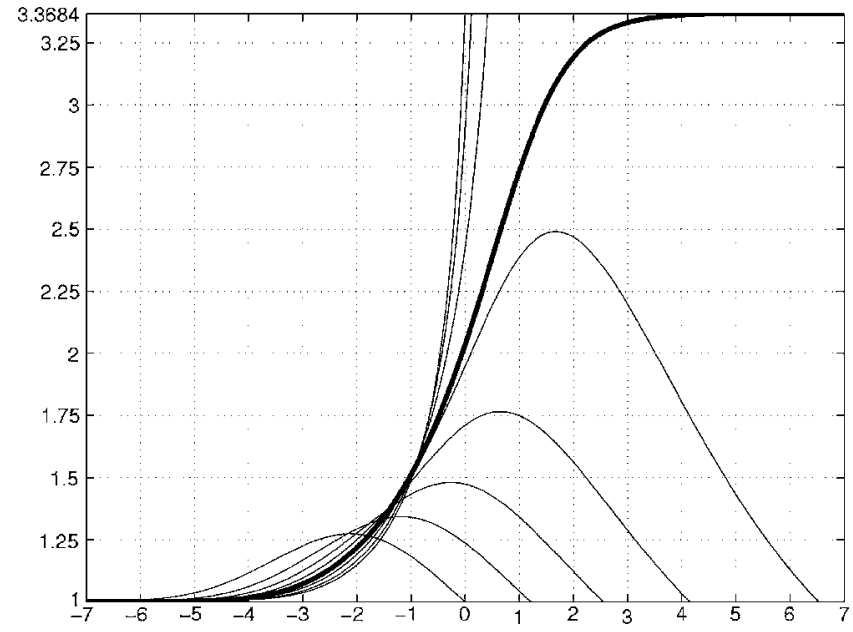

FIG. 1. Family of normalized $\rho(x)$ obtained from the numerical solution of Eqs. (10) and (11) in $\mathrm{Ma}=4$ argon shocks for different values $\rho_{C}$. Argon parameters: $\gamma=5 / 3, \operatorname{Pr}=2 / 3, \omega=0.81$ (Ref. 1). The bold line is the converged solution. See Ref. 8 for normalization.

The thermal dependence of the bulk viscosity $\xi$ will be commented below in some detail.

We present next some details of the numerical technique employed, which is not original, but contains some nonobvious mathematical features. Integration of the system (1)-(3) leads to

$$
\begin{aligned}
& \rho u=c_{0}, \\
& \rho u^{2}+p=\Pi+c_{1}, \\
& \rho u H+q=\Pi u+c_{2},
\end{aligned}
$$

where $c_{0}, c_{1}, c_{2}$ are constants. Using the quantities defined above, the system (7)-(9) may be reduced to two equations for $\rho$ and $p$,

$$
\begin{aligned}
\frac{d \rho}{d x}= & \frac{\rho^{2}}{\left(\frac{4}{3} \eta+\xi\right) c_{0}}\left(c_{1}-p-\frac{c_{0}^{2}}{\rho}\right), \\
\frac{d p}{d x}= & \frac{d \rho}{d x} \cdot \frac{p}{\rho}-\frac{R \rho}{\kappa}\left(c_{2}-\left(\frac{4}{3} \eta+\xi\right) \frac{c_{0}^{2}}{\rho^{3}} \frac{d \rho}{d x}-\frac{c_{0}^{3}}{2 \rho^{2}}\right. \\
& \left.-\frac{c_{0} p}{\rho} \cdot \frac{\gamma}{\gamma-1}\right) .
\end{aligned}
$$

The problem consists of finding the solution of the system (10) and (11) with the asymptotic left $(L)$ and right $(R)$ Rankine-Hugoniot boundary conditions, referred to in Fig. 1,

$$
\rho_{R}=\rho_{L} \frac{(\gamma+1) \mathrm{Ma}^{2}}{2+(\gamma-1) \mathrm{Ma}^{2}}, \quad p_{R}=p_{L} \frac{2 \gamma \mathrm{Ma}^{2}-(\gamma-1)}{(\gamma+1)},
$$

where $\mathrm{Ma}=u_{L} / \sqrt{\gamma R T_{L}}$.

The constants in the system (10) and (11) are derived from the boundary conditions, becoming

$$
c_{0}=\mathrm{Ma} \sqrt{\gamma p_{L} \rho_{L}}, \quad c_{1}=\gamma p_{L}\left(\mathrm{Ma}^{2}+\frac{1}{\gamma}\right),
$$

$$
c_{2}=\gamma p_{L} \sqrt{\frac{\gamma p_{L}}{\rho_{L}}}\left(\frac{\mathrm{Ma}^{3}}{2}+\frac{\mathrm{Ma}}{\gamma-1}\right) .
$$

The numerical solution of the system (10) and (11) with the boundary conditions (12) pose two difficulties:

If two functions $\rho(x)$ and $p(x)$ are a solution of the system, any two functions $\widetilde{\rho}(x)=\rho(x+c), \widetilde{p}(x)=p(x$ $+c$ ), where $c$ is an arbitrary constant: is also a solution of the system, i.e., the solution of the system is not unique, existing of a family of solutions;

additional difficulties arise from the asymptotic boundary conditions.

The problem is solved by means of the following algorithm. Let us choose in the central point $x_{C}=0$ an additional condition $p\left(x_{C}\right)=\left(p_{R}+p_{L}\right) / 2$. So we assure the unique solution for (i), and a nonasymptotic condition for (ii). Taking $\rho\left(x_{C}\right)=\rho_{C}$ as a parameter, for every chosen value of $\rho_{C}$ we have an initial-value problem (Cauchy problem) for the system of two equations with two initial conditions in the point $x_{C}$. The system is solved by the Runge-Kutta method to the left and to the right from some fixed point $x_{C}$. The unknown value of the parameter $\rho_{C}$ is determined iteratively by solving a number of such problems, satisfying $L$ and $R$ asymptotic boundary conditions (12).

As an example Fig. 1 shows the sequence of iterations for different values of $\rho_{C}$ corresponding to a $\mathrm{Ma}=4$ shock in argon. All $\rho(x)$ 's satisfy the $L$ asymptotic boundary condition, but only the true solution satisfies both $L$ and $R$ conditions. The solutions so obtained are very sensitive to parameter $\rho_{C}$, and the deflection of the trial solutions from the true one is maximal at the $R$ boundary. The converged value of parameter $\rho_{C}$ was obtained by successive use of the simple halving method (dichotomy method). The $R$-boundary condition was fulfilled with a relative accuracy of $\Delta \rho / \rho<10^{-2}$, corresponding to the uncertainty of the parameter $\Delta \rho_{C} / \rho_{C}$ $<10^{-7}$. So one obtains a function $\rho(x)$, which differs from the exact value at point $x_{C}$ by less than the quoted uncertainty. From Eqs. (10) and (11) it is clear that the same accuracy is obtained for the derivatives $d \rho / d x, d p / d x$ at the center of the $R-L$ interval. Other details of the numerical algorithm have been described in Ref. 10.

As an accuracy test the reciprocal shock thickness

$$
1 / \delta=\max \left(\frac{d \rho}{d x}\right) \frac{1}{\rho_{R}-\rho_{L}}
$$

is determined for $1.5<\mathrm{Ma}<10$ shocks in argon, where $\xi$ $=0$. Agreement to $1 \%$ is found with a previous work where the same problem was solved by a finite-difference timeevolution method. ${ }^{8}$ This ensures the accuracy of both methods and the numerical results for argon shocks. The relaxation method proves, however, much less efficient for the $\mathrm{N}_{2}$ shocks. While the present method yields a converged solution in $\approx 20 \mathrm{sec}$ for several values in $1.5<\mathrm{Ma}<10$ with accuracy $10^{-7}$, the above-mentioned relaxation method took several hours for a single point $(\mathrm{Ma}=7)$ with accuracy 0.001 . The reason can be easily inferred from Ref. 8 . 
Diatomic molecules have two rotational degrees of freedom which shall be considered in the frame of the present problem by means of a temperature-depending bulk viscosity, which is specific of the molecular species. According to Ref. 11 the bulk viscosity coefficient for a gas of molecules with rotational degrees of freedom is given by

$$
\xi=\frac{p R}{c_{V}} \gamma_{\text {rot }} \tau_{\text {rot }},
$$

where $c_{V}=R /(\gamma-1)$ is the heat capacity at constant volume, and

$$
\gamma_{\text {rot }}=\frac{3}{2}\left(\frac{5}{3}-\gamma\right) .
$$

is the contribution to the heat capacity ratio due to the rotational degrees of freedom. The relaxation time $\tau_{\text {rot }}$ may be expressed as $\tau_{\text {rot }}=Z \tau_{c}$ in terms of the collision number $Z$ and of the mean time between collisions, $\tau_{c}=\lambda / \sqrt{8 R T / \pi}$. According to Ref. 1 the molecule-specific mean free path is

$$
\lambda=\frac{2(7-2 \omega)(5-2 \omega)}{15 \sqrt{2 \pi}} \frac{\eta}{p} \sqrt{R T} .
$$

The above relations lead to a bulk viscosity of the form

$$
\xi=\eta\left(\frac{5}{3}-\gamma\right) B,
$$

where

$$
B=Z(\gamma-1) \frac{(7-2 \omega)(5-2 \omega)}{20}
$$

is a dimensionless coefficient.

The collision number $Z$ has been modeled according to Parker $^{12}$ as

$$
Z=\frac{Z_{\infty}}{1+\left(\pi^{3 / 2} / 2\right)\left(T^{*} / T\right)^{1 / 2}+\left(\pi+\pi^{2} / 4\right)\left(T^{*} / T\right)},
$$

with $Z_{\infty}=23, T^{*}=91.5 \mathrm{~K}$ for molecular nitrogen. ${ }^{1}$ The other physical parameters for molecular nitrogen were ${ }^{1} \gamma=7 / 5$, $\omega=0.74, \operatorname{Pr}=14 / 19$. Here $\omega$ is taken in its simplest form, as a constant, in order to show more clearly the effect of the bulk viscosity compared with previous NS calculations. However, the method used permits to employ $\omega=\omega(T)$.

The system of equations (7)-(9) is solved by the method outlined above, employing the shear-stress tensor defined by (4), including the bulk viscosity (16).

The comparison with the experimental data compiled in Ref. 4 is shown in Figs. 2 and 3, normalized with the mean free-path for argon at the $L$ boundary of the shock wave. Calculated for hard-sphere molecules $(\omega=0.5)$ as

$$
\lambda_{L}=\frac{16}{5} \frac{\eta_{L}}{\rho_{L} \sqrt{2 \pi R T_{L}}},
$$

it yields $\lambda_{\mathrm{Ar}}=1.098 \mathrm{~mm}$ for $p_{L}=50 \mathrm{mTorr}$ and $T_{L}=300 \mathrm{~K}$. In these conditions $\lambda_{\mathrm{Ar}} / \lambda_{\mathrm{N}_{2}}=\eta_{\mathrm{Ar}} / \eta_{\mathrm{N}_{2}} \sqrt{m_{\mathrm{Ar}} / m_{\mathrm{N}_{2}}}=1.060$. This very same mean-free-path definition was employed for the normalization of experimental shock wave thickness in Ref. 4.

The computational results are summarized in Fig. 2: line 1 refers to the calculation ignoring the contribution of bulk

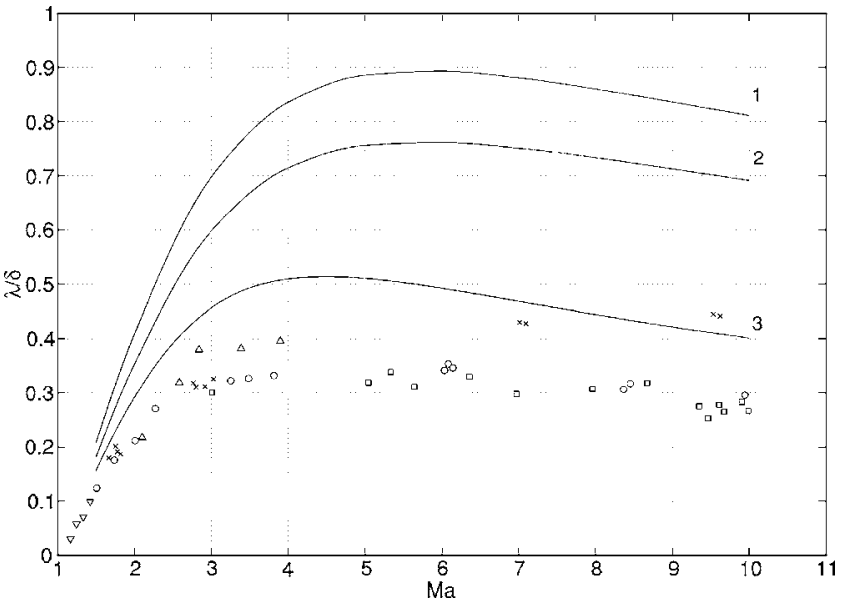

FIG. 2. Reciprocal shock thickness $\lambda_{L} / \delta$ vs Mach number for nitrogen in comparison with experiments (markers). Lines: calculations for (1) $B=0,(2)$ $B=1$, and (3) $B=B(Z, T)$.

viscosity $(B=0)$; line 2 corresponds to the simplest form of bulk viscosity $(B=1)$; line 3 is the solution for $B$ defined by (17), varying within $1<B<3$ for the temperatures corresponding to the shock waves with $1.5<\mathrm{Ma}<10$. It is clear that including the bulk viscosity in the calculation reduces the difference with the experiment to $\approx 30 \%$.

The calculated density, velocity, and temperature profiles of nitrogen shock waves with $\mathrm{Ma}=6.1$ are shown in Fig. 3 jointly with the experimental density profiles from Ref. 4. These results confirm the strong influence of the bulk viscosity, showing that the model described by Eqs. (16) and (17) yields a density profile very close to the experiment.

The lag between density and temperature shock profiles of Fig. 3 is qualitatively consistent with previous studies, but the overshoot of the temperature profile predicted by a more elaborate model based, for example, on the quasi-gasdynamic equations with translational-rotational nonequilibrium, ${ }^{13,14}$ is not accounted for by the present model.

Present results, and the previous ones on argon shock waves, ${ }^{8}$ show, however, the reasonable sufficiency of the NS equations to account for the density profile of shock waves

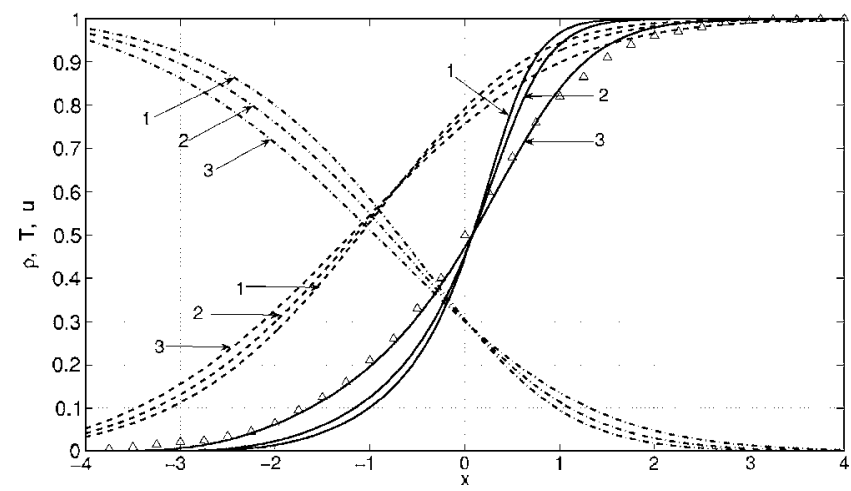

FIG. 3. Normalized density (line), velocity (dash-dot), and temperature (dashed line) profiles of a $\mathrm{Ma}=6.1$ shock in nitrogen, compared with experiment (markers). Lines: calculations for (1) $B=0$, (2) $B=1$, and (3) $B$ $=B(Z, T)$. See Ref. 8 for normalization. 
up to Mach number much higher than supposed before. An agreement of $\approx 30 \%$ is attained for the $\mathrm{N}_{2}$ shock wave thickness in the $1.5<\mathrm{Ma}<10$ regime employing a manageable version of the bulk viscosity. Nonetheless, much work remains to be done, especially in the domain of temperature shock profiles, where the validity of the NS approach has not been confirmed so far, and where the experimental data are lacking. In any case, a detailed description of the bulk viscosity in terms of the collisional regime appears a necessary requirement towards the accurate description of the shock wave profiles in the NS approach.

${ }^{1}$ G. A. Bird, Molecular Gas Dynamics and the Direct Simulation of Gas Flows (Clarendon, Oxford, 1998).

${ }^{2}$ C. Cercigniani, Rarefied Gas Dynamics (Cambridge University Press, Cambridge, 2000).

${ }^{3}$ M. N. Kogan, Rarefied Gas Dynamics (Plenum, New York, 1969).

${ }^{4} \mathrm{H}$. Alsmeyer, "Density profiles in argon and nitrogen shock waves measured by the absorption of an electron beam," J. Fluid Mech. 74, 497 (1976).

${ }^{5}$ L. M. Schwartz and D. F. Hornig, "Navier-Stokes calculations of argon shock wave structure," Phys. Fluids 6, 1669 (1963).
${ }^{6}$ M. Linzer and D. F. Hornig, "Structure of shock fronts in argon and nitrogen," Phys. Fluids 6, 1661 (1963).

${ }^{7}$ M. Torrilhon and H. Struchtrup, "Regularized 13-moment equations: shock structure calculations and comparison to Burnett models," J. Fluid Mech. 513, 171 (2004).

${ }^{8}$ T. G. Elizarova, I. A. Shirokov, and S. Montero, "Numerical simulation of shock wave structure for argon and helium," Phys. Fluids 17, 068101 (2005).

${ }^{9}$ J. D. Lambert, Vibrational and Rotational Relaxation in Gases (Oxford University Press, Oxford, 1977).

${ }^{10}$ T. G. Elizarova and A. A. Khokhlov, "Numerical simulation of shockwave structure by solving stationary Navier-Stokes equations," Moscow Univ. Phys. Bull. 3, 28 (2006).

${ }^{11}$ V. M. Zdanov and M. Ya. Alievskii, Transport and Relaxation Processes in Molecular Gases (Nauka, Moscow, 1989).

${ }^{12}$ J. G. Parker, "Rotational and vibrational relaxation in diatomic gases," Phys. Fluids 2, 449 (1959).

${ }^{13}$ T. G. Elizarova and I. A. Chirokov, "Macroscopic model for gas with translational-rotational nonequilibrium," Comput. Math. Math. Phys. 39, 135 (1999) [Zh. Vychisl. Mat. Mat. Fiz. 39, 141 (1999)].

${ }^{14}$ I. A. Chirokov, T. G. Elizarova, and J. C. Lengrand, "Numerical study of shock wave structure based on quasigasdynamic equations with rotational nonequilibrium," in Proceedings of the 21st International Symposium on Rarefied Gas Dynamics, 26-31 July 1998, Marseille, France, edited by R. Brun et al. (Cepadues, Toulouse, France, 1999), Vol. 1, pp. 175-182. 